\title{
Clinical characteristics and outcomes of paediatric orbital cellulitis in Hospital Universiti Sains Malaysia: a five-year review
}

\author{
Ismail Mohd-Ilham ${ }^{1,2}$, MBBS, MMed, Abd Bari Muhd-Syafi' ${ }^{1,2}$, MBBS, Sonny Teo Khairy-Shamel ${ }^{1,2}$, MD, MMed, \\ Ismail Shatriah $^{1,2}$, MD, MMed
}

\begin{abstract}
INTRODUCTION Limited data is available on paediatric orbital cellulitis in Asia. We aimed to describe demographic data, clinical presentation, predisposing factors, identified microorganisms, choice of antibiotics and management in children with orbital cellulitis treated in a tertiary care centre in Malaysia.

METHODS A retrospective review was performed on children with orbital cellulitis aged below 18 years who were admitted to Hospital Universiti Sains Malaysia, Kelantan, Malaysia, between January 2013 and December 2017.

RESULTS A total of 14 paediatric patients fulfilling the diagnostic criteria for orbital cellulitis were included. Their mean age was $6.5 \pm 1.2$ years. Boys were more likely to have orbital cellulitis than girls $(71.4 \%$ vs. $28.6 \%)$. Involvement of both eyes was observed in $14.3 \%$ of the patients. Sinusitis $(28.6 \%)$ and upper respiratory tract infection $(21.4 \%)$ were the most common predisposing causes. Staphylococcus aureus (28.6\%) was the leading pathogen. Longer duration of hospitalisation was observed in those infected with methicillin-resistant Staphylococcus aureus and Burkholderia pseudomallei. 10 (71.4\%) patients were treated with a combination of two or three antibiotics. In this series, $42.9 \%$ had surgical interventions.

CONCLUSION Young boys were found to be more commonly affected by orbital cellulitis than young girls. Staphylococcus aureus was the most common isolated microorganism. Methicillin-resistant Staphylococcus aureus and Burkholderia pseudomallei caused severe infection. Sinusitis and upper respiratory tract infection were the most common predisposing factors. A majority of the children improved with medical treatment alone. Our findings are in slight disagreement with other published reports on paediatric orbital cellulitis, especially from the Asian region.
\end{abstract}

Keywords: Asian children, orbital abscess, orbital cellulitis

\section{INTRODUCTION}

Orbital cellulitis in children is a serious infection that can result in significant complications including blindness, cavernous sinus thrombosis, meningitis, subdural empyema and brain abscess. ${ }^{(1-3)}$ Prompt diagnosis and early treatment are important to prevent potential vision or life-threatening conditions.

Numerous reports on paediatric orbital cellulitis have been published in the literature. ${ }^{(4-12)}$ Despite the abundance of reports on the topic, among Asian countries, data was only available from Singapore, India and Taiwan. ${ }^{(12-15)}$ These series included data on both adults and children. ${ }^{(13-15)}$ In this study, we described demographics, clinical characteristics, causative organisms, treatment and visual outcomes among paediatric orbital cellulitis cases treated in Malaysia, and reviewed the relevant literature.

\section{METHODS}

A retrospective case series was conducted on all children aged under 18 years who were diagnosed with orbital cellulitis and admitted to Hospital Universiti Sains Malaysia, Kelantan, Malaysia. Data was collected from 1 January 2013 till 31 December 2017. The study was conducted in accordance with the Declaration of Helsinki.
Cases were identified through a database search of medical records using the International Classification of Diseases Ninth and Tenth Revision codes (376.01, H05.0) for orbital cellulitis. A total of 44 paediatric patients with the diagnosis of orbital cellulitis were identified on the admission computers, and their medical records were retrieved. Children who were diagnosed with orbital cellulitis and admitted to the ward were included in our study. We excluded those who had pre-existing ocular diseases such as corneal opacity, congenital cataracts, strabismus, refractive errors, optic disc or macular problems. A final list was reviewed, and 14 patients were carefully selected. Data documented included age, gender, laterality, possible identified cause of infection, presenting clinical manifestations, imaging results, microbiology results, treatment options (medical treatment alone or in combination with surgical drainage), length of hospital stay and final visual outcome. The institution required patients' paediatric orbital cellulitis registry forms to be completed by a consultant paediatric ophthalmologist. Data was analysed using SPSS Statistics version 22.0 (IBM Corp, Armonk, NY, USA).

\section{RESULTS}

A total of 14 paediatric patients with orbital cellulitis in 16 eyes were recruited according to the study protocol. Table I summarises

${ }^{1}$ Department of Ophthalmology, School of Medical Sciences, Universiti Sains Malaysia, ${ }^{2}$ Hospital Universiti Sains Malaysia, Kelantan, Malaysia Correspondence: Dr Ismail Shatriah, Consultant Paediatric Ophthalmologist, Department of Ophthalmology, School of Medical Sciences, Universiti Sains Malaysia, 16150 Kubang Kerian, Kelantan, Malaysia. shatriah@usm.my 
Table I. Summary of clinical cases $(n=14)$.

\begin{tabular}{|c|c|c|c|c|c|c|c|c|c|}
\hline No. & $\begin{array}{l}\text { Age; } \\
\text { gender }\end{array}$ & $\begin{array}{l}\text { Clinical presentation; } \\
\text { onset; laterality }\end{array}$ & CT result & Cause & Culture; investigation & Antibiotic (duration) & Surgery & Hospital LOS & $\begin{array}{l}\text { Presenting } \\
\text { VA; final VA }\end{array}$ \\
\hline 1 & $6 \mathrm{yr} ; \mathrm{M}$ & $\begin{array}{l}\text { Lid swelling, ptosis, } \\
\text { high-grade fever; } 3 \text { days; } \\
\text { right eye }\end{array}$ & $\begin{array}{l}\text { Extraconal abscess } \\
\text { (subperiosteal } \\
\text { abscess) }\end{array}$ & $\begin{array}{l}\text { Upper respiratory } \\
\text { tract infection and } \\
\text { sinusitis }\end{array}$ & $\begin{array}{l}\text { No growth from eye swab, } \\
\text { blood and urine samples; } \\
\text { TWC 10.6, PLT 383, ESR 96, } \\
\text { CRP NA }\end{array}$ & $\begin{array}{l}\text { IV ceftriaxone ( } 1 \text { wk), } \\
\text { IV Augmentin ( } 5 \text { days) }\end{array}$ & Nil & 12 days & $6 / 6 ; 6 / 6$ \\
\hline 2 & $5 \mathrm{yr} ; \mathrm{M}$ & $\begin{array}{l}\text { Lid swelling, erythema, } \\
\text { ptosis, high-grade fever, } \\
\text { intracranial abscess; } \\
7 \text { days; left eye }\end{array}$ & $\begin{array}{l}\text { Lid and cerebral } \\
\text { abscess }\end{array}$ & $\begin{array}{l}\text { Trauma - hit by } \\
\text { toys, underlying } \\
\text { sinusitis }\end{array}$ & $\begin{array}{l}\text { No growth from pus, } \\
\text { blood and urine samples; } \\
\text { TWC 14.7, PLT 444, ESR } \\
\text { 106, CRP positive }\end{array}$ & $\begin{array}{l}\text { IV ceftriaxone } \\
(7 \mathrm{wk}), \text { IV } \\
\text { metronidazole } \\
(6 \mathrm{wk})\end{array}$ & $\begin{array}{l}\text { Open lid incision } \\
\text { and drainage }\end{array}$ & 7 wk & $\begin{array}{l}\text { Not } \\
\text { cooperative; } \\
6 / 6\end{array}$ \\
\hline 3 & $2 \mathrm{yr} ; \mathrm{M}$ & $\begin{array}{l}\text { Lid swelling, ptosis, } \\
\text { chemosis, high-grade } \\
\text { fever; } 2 \text { days; both eyes }\end{array}$ & $\begin{array}{l}\text { Medial canthus } \\
\text { abscess }\end{array}$ & $\begin{array}{l}\text { Upper respiratory } \\
\text { tract infection } \\
\text { and exudative } \\
\text { tonsillopharyngitis }\end{array}$ & $\begin{array}{l}\text { Streptococcus pneumoniae } \\
\text { (pus); TWC 19.6, PLT 271, } \\
\text { ESR NA, CRP positive }\end{array}$ & $\begin{array}{l}\text { IV Augmentin } \\
(5 \text { days), IV cloxacillin } \\
\text { ( } 5 \text { days) }\end{array}$ & $\mathrm{Nil}$ & $1 \mathrm{wk}$ & $\mathrm{NA}$; NA \\
\hline 4 & $6 \mathrm{yr} ; \mathrm{M}$ & $\begin{array}{l}\text { Lid swelling, ptosis, } \\
\text { chemosis; } 1 \text { day; right } \\
\text { eye }\end{array}$ & Lid abscess & $\begin{array}{l}\text { No identified } \\
\text { cause }\end{array}$ & $\begin{array}{l}\text { No growth from eye swab, } \\
\text { blood and urine samples; } \\
\text { TWC NA, ESR NA, CRP NA }\end{array}$ & $\begin{array}{l}\text { IV Augmentin } \\
\text { ( } 5 \text { days) }\end{array}$ & Nil & 5 days & $6 / 9 ; 6 / 6$ \\
\hline 5 & $14 \mathrm{yr} ; \mathrm{M}$ & $\begin{array}{l}\text { Lid swelling, ptosis, } \\
\text { chemosis, low-grade } \\
\text { fever; } 1 \text { day; right eye }\end{array}$ & $\begin{array}{l}\text { Periorbital tissue } \\
\text { thickening } \\
\text { extending to right } \\
\text { temporal region }\end{array}$ & $\begin{array}{l}\text { Trauma and } \\
\text { laceration wound } \\
\text { at the temporal } \\
\text { eyelid }\end{array}$ & $\begin{array}{l}\text { Streptococcus beta- } \\
\text { haemolytic group A; } \\
\text { Staphyloccus aureus (pus); } \\
\text { TWC 32.6, PLT 150, ESR } \\
\text { NA, CRP NA }\end{array}$ & $\begin{array}{l}\text { IV ceftriaxone } \\
(2 w k) \text {, IV cloxacillin } \\
(2 w k)\end{array}$ & $\begin{array}{l}\text { Open lid incision } \\
\text { and drainage }\end{array}$ & $2 w k$ & $6 / 6 ; 6 / 6$ \\
\hline 6 & $13 \mathrm{yr} ; \mathrm{M}$ & $\begin{array}{l}\text { Lid swelling, chemosis, } \\
\text { low-grade fever; } 5 \text { days; } \\
\text { right eye }\end{array}$ & Not done & $\begin{array}{l}\text { Upper respiratory } \\
\text { tract infection }\end{array}$ & $\begin{array}{l}\text { No growth from eye swab, } \\
\text { blood and urine samples; } \\
\text { TWC 7.6, PLT 206, ESR NA, } \\
\text { CRP NA }\end{array}$ & $\begin{array}{l}\text { IV Augmentin } \\
\text { (5 days) }\end{array}$ & Nil & 6 days & $6 / 12 ; 6 / 6$ \\
\hline 7 & $\begin{array}{l}39 \text { days; } \\
\text { M }\end{array}$ & $\begin{array}{l}\text { Facial swelling, proptosis, } \\
\text { chemosis, high-grade } \\
\text { fever; } 1 \text { day; right eye }\end{array}$ & $\begin{array}{l}\text { Periorbital and } \\
\text { facial abscess }\end{array}$ & Gingivostomatitis & $\begin{array}{l}\text { Methicillin-resistant } \\
\text { Staphylococous aureus } \\
\text { (from eye swab and blood } \\
\text { sample); TWC 13.4, PLT } \\
\text { 511, ESR NA, CRP positive }\end{array}$ & $\begin{array}{l}\text { IV ceftazidime } \\
\text { (14 days), IV } \\
\text { metronidazole } \\
\text { (14 days), IV } \\
\text { vancomycin (10 days) }\end{array}$ & $\begin{array}{l}\text { Image-guided } \\
\text { endoscopic } \\
\text { drainage }\end{array}$ & 23 days & $\mathrm{NA} ; \mathrm{NA}$ \\
\hline 8 & $10 \mathrm{yr} ; \mathrm{F}$ & $\begin{array}{l}\text { Lid swelling, chemosis, } \\
\text { high-grade fever; } 3 \text { days; } \\
\text { left eye }\end{array}$ & $\begin{array}{l}\text { Soft tissue } \\
\text { thickening and } \\
\text { lacrimal sac abscess }\end{array}$ & Dacryocystitis & $\begin{array}{l}\text { No growth from eye swab, } \\
\text { blood and urine samples; } \\
\text { TWC 12.01, PLT 270, ESR } \\
\text { NA, CRP NA }\end{array}$ & $\begin{array}{l}\text { IV Augmentin } \\
\text { ( } 5 \text { days), IV } \\
\text { ceftazidime ( } 1 \mathrm{wk}) \text {, IV } \\
\text { metronidazole ( } 1 \mathrm{wk})\end{array}$ & Nil & 11 days & $6 / 6 ; 6 / 6$ \\
\hline 9 & $6 \mathrm{yr} ; \mathrm{M}$ & $\begin{array}{l}\text { Lid swelling, chemosis, } \\
\text { ophthalmoplegia, low- } \\
\text { grade fever; } 1 \text { day; left eye }\end{array}$ & $\begin{array}{l}\text { Lid abscess and } \\
\text { orbital cellulitis }\end{array}$ & Pansinusitis & $\begin{array}{l}\text { No growth from eye swab, } \\
\text { blood and urine samples; } \\
\text { TWC 29.7, PLT 896, ESR } \\
\text { NA, CRP NA }\end{array}$ & $\begin{array}{l}\text { IV Augmentin } \\
\text { ( } 5 \text { days), IV ceftazidime } \\
\text { ( } 5 \text { days), IV } \\
\text { metronidazole ( } 5 \text { days) }\end{array}$ & $\begin{array}{l}\text { Nasal decongestion } \\
\text { and left antral } \\
\text { washout }\end{array}$ & 8 days & $\begin{array}{l}\text { Not } \\
\text { cooperative; } \\
6 / 9\end{array}$ \\
\hline 10 & $11 \mathrm{yr} ; \mathrm{F}$ & $\begin{array}{l}\text { Lid swelling, chemosis, } \\
\text { no fever; } 7 \text { days; left eye }\end{array}$ & Not done & $\begin{array}{l}\text { Infected wound } \\
\text { from a ruptured } \\
\text { upper lid abscess }\end{array}$ & $\begin{array}{l}\text { Staphylococcus aureus } \\
\text { (pus); TWC 9.9, PLT 263, } \\
\text { ESR NA, CRP NA }\end{array}$ & $\begin{array}{l}\text { IV Augmentin } \\
\text { ( } 5 \text { days) }\end{array}$ & Nil & 7 days & $6 / 21 ; 6 / 6$ \\
\hline
\end{tabular}




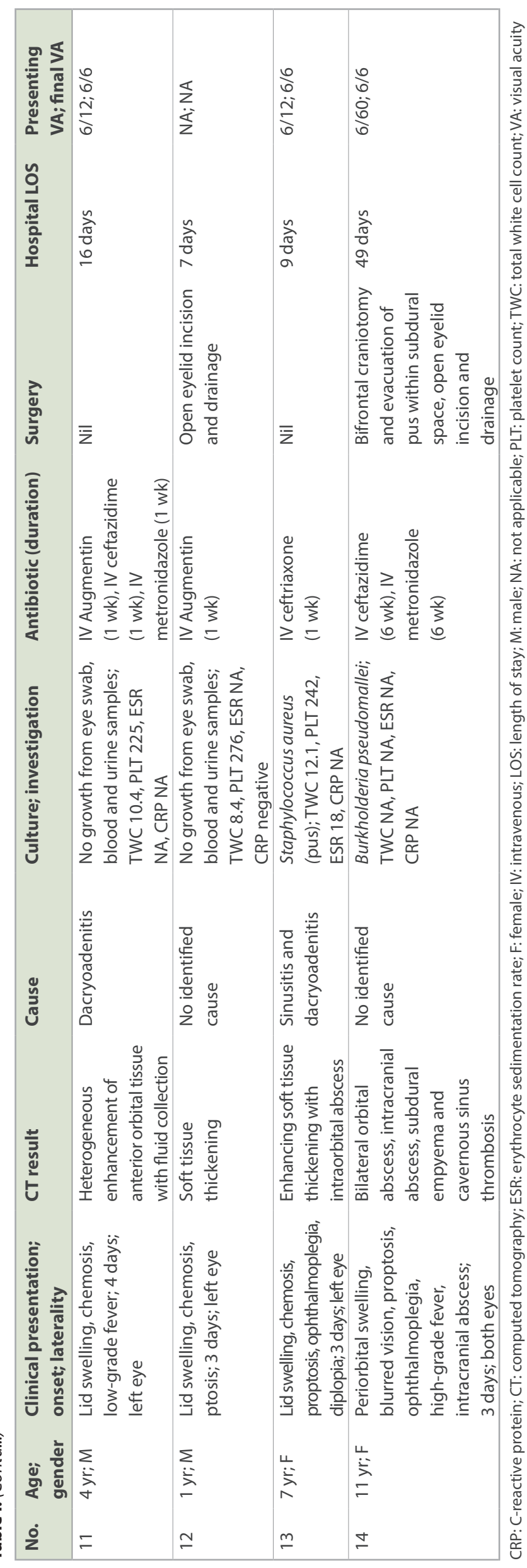

the clinical profile of all patients involved in this study. The mean age of the patients was $6.5 \pm 1.2$ years. The majority were male (11 eyes, 68.8\%). 7 (50.0\%) patients had left eye involvement and $2(14.3 \%)$ patients had bilateral involvement.

Lid swelling and conjunctival chemosis were the most common presenting features $(100.0 \%$ and $78.6 \%$, respectively). $5(35.7 \%)$ patients had presenting visual acuity of 6/12 (20/40) and worse. $11(78.6 \%)$ patients had final visual acuity of 6/9 (20/32) or better. The final visual acuity of three young patients aged 39 days, one year and two years was not documented, as they were uncooperative during the examination. The mean duration of hospitalisation was $13.2 \pm 11.9$ days (Table II).

$6(42.9 \%)$ patients had been treated with oral antibiotics prior to referral to the centre. The most commonly prescribed antibiotics were Augmentin and ceftriaxone. Other antibiotics included metronidazole, cloxacillin, ceftazidime and/or vancomycin. The majority of the patients $(71.4 \%, \mathrm{n}=10)$ received intravenous antibiotics for seven days or less, with 2 (14.3\%) patients receiving antibiotics for six weeks due to intracranial complications.

The most commonly isolated organisms were Staphylococcus species in $4(28.6 \%)$ patients and Streptococcus species in $2(14.3 \%)$ patients. Blood cultures were negative for all patients, except for one patient aged 39 days with methicillin-resistant Staphylococcus aureus. All patients were promptly treated with intravenous antibiotics upon admission. 8 (57.1\%) patients were successfully treatment with medical treatment alone, while $6(42.9 \%)$ patients were treated with a combination of medical treatment and surgical interventions.

\section{DISCUSSION}

There have been limited reviews of paediatric orbital cellulitis in Asian countries. ${ }^{(12-15)}$ Yang et al described a report involving 20 children with orbital cellulitis treated in Singapore, ${ }^{(12)}$ while Liu et al, Babu et al and Pandian et al reported data from Taiwan ${ }^{(13,14)}$ and India, ${ }^{(15)}$ respectively, but their inclusion criteria were slightly different from those in this study. Table II summarises published reports on paediatric orbital cellulitis from Asian countries, Australia, the Middle East, United Kingdom, Scotland, Canada and the United States, including data from the present study. ${ }^{(4-7,9,12,13,15)}$

The mean age of presentation of our patients was $6.5 \pm 1.2$ years, which was consistent with other published reports. ${ }^{(5-7,12)}$ $71.4 \%$ of our patients were boys, a similar observation to the reports of Ferguson et al and Nageswaran et al. ${ }^{(4,5)}$ In contrast, a Scotland study of 243 children found that slightly more girls than boys had orbital cellulitis. ${ }^{(6)}$

Left eye involvement was observed in 50\% of our patients. A higher percentage of left eye involvement was also documented by Nageswaran et al $(56.1 \%),{ }^{(5)}$ Fanella et al $(65.8 \%),{ }^{(7)}$ and Atfeh and Khalil $(61.0 \%) .{ }^{(9)}$ In our study, both eyes were involved in $2(14.3 \%)$ patients. One of the two patients had an abscess in the medial canthal area, while the second developed severe septicaemia. Bilateral eye involvement was also reported by Nageswaran et al $(34.1 \%)^{(5)}$ as well as Atfeh and Khalil (4.0\%). ${ }^{(9)}$ 
Original Article

Table II. Comparison of published studies on paediatric orbital cellulitis.

\begin{tabular}{|c|c|c|c|c|c|c|c|c|c|}
\hline \multirow[t]{2}{*}{ Variable } & \multicolumn{9}{|c|}{ No. $(\%) / m e a n \pm$ standard deviation } \\
\hline & $\begin{array}{l}\text { Present } \\
\text { study }\end{array}$ & Yang et al $\left.\right|^{(12)}$ & Liu et al ${ }^{(15)}$ & Babu et al ${ }^{(13)}$ & $\begin{array}{l}\text { Ferguson } \\
\text { et al }{ }^{(4)}\end{array}$ & Atfeh \& Khalil(9) & Crosbie et al(6) & $\begin{array}{l}\text { Nageswaran } \\
\text { et } \text { al }^{(5)}\end{array}$ & $\begin{array}{l}\text { Fanella } \\
\text { et al }\left.\right|^{(7)}\end{array}$ \\
\hline Country; yr & $\begin{array}{l}\text { Malaysia; } \\
2020\end{array}$ & $\begin{array}{l}\text { Singapore; } \\
2009\end{array}$ & Taiwan; 2006 & India; 2016 & $\begin{array}{l}\text { Australia; } \\
1999\end{array}$ & $\begin{array}{l}\text { United Kingdom; } \\
2015\end{array}$ & Scotland; 2016 & $\begin{array}{l}\text { United States; } \\
2006\end{array}$ & $\begin{array}{l}\text { Canada; } \\
2011\end{array}$ \\
\hline Population & 14 children & 20 children & $\begin{array}{l}\text { Total } 27 \\
\text { ( } 8 \text { children } \\
+ \text { adults })\end{array}$ & $\begin{array}{l}\text { Total } 14 \\
(5 \text { children }+ \\
\text { adults) }\end{array}$ & $\begin{array}{l}\text { Total } 52(34 \\
\text { children) }+ \\
\text { adults })\end{array}$ & $\begin{array}{l}\text { Total } 54(40 \\
\text { children } \\
+ \text { adults })\end{array}$ & $\begin{array}{l}\text { Total } 234 \text { (including } 30 \\
\text { children who fulfilled } \\
\text { Chandler classification) }\end{array}$ & 41 children & $\begin{array}{l}38 \\
\text { children }\end{array}$ \\
\hline Age (yr) & $6.5 \pm 1.2$ & 5.5 & $0-18$ & NA & NA & $5.8 \pm 4.5$ & $4.7 \pm 4.4$ & 7.5 & 7.5 \\
\hline Gender & & & $M>F$ & & & & & & \\
\hline Male & $10(71.4)$ & NA & & $2(40.0)$ & 25 (73.5) & NA & (51.9) & 30 (73.2) & NA \\
\hline Female & $4(28.6)$ & NA & & $3(60.0)$ & $9(26.5)$ & NA & $(48.1)$ & $11(26.8)$ & NA \\
\hline \multicolumn{10}{|l|}{ Laterality } \\
\hline Right & $5(35.7)$ & NA & NA & $3(60.0)$ & NA & (35.0) & NA & $4(9.8)$ & $13(34.2)$ \\
\hline Left & $7(50.0)$ & NA & NA & $2(40.0)$ & NA & $(61.0)$ & NA & $23(56.1)$ & $25(65.8)$ \\
\hline Both & $2(14.3)$ & NA & NA & $0(0)$ & NA & $(4.0)$ & NA & $14(34.1)$ & $0(0)$ \\
\hline Antibiotics before admission & $6(42.9)$ & NA & NA & NA & $11(32.4)$ & NA & NA & $33(80.5)$ & $22(57.9)$ \\
\hline Fever on/after presentation $\left(>38^{\circ} \mathrm{C}\right)$ & $6(42.9)$ & $20(100.0)$ & NA & NA & $9(26.5)$ & NA & NA & $27(65.9)$ & NA \\
\hline TWC $>15,000$ & $3(21.4)$ & $13(65.0)$ & NA & NA & NA & NA & NA & $19(46.3)$ & $13(34.2)$ \\
\hline \multicolumn{10}{|l|}{ Predisposing factors } \\
\hline Sinusitis & $4(28.6)$ & $6(30.0)$ & NA & $4(80.0)$ & $31(91.2)$ & NA & NA & NA & $36(94.7)$ \\
\hline Diabetes mellitus & $0(0)$ & $0(0)$ & NA & $1(20.0)$ & $0(0)$ & NA & NA & NA & NA \\
\hline \multicolumn{10}{|l|}{ Other } \\
\hline Dental abscess & $1(7.1)$ & $0(0)$ & NA & NA & $1(2.9)$ & NA & NA & NA & NA \\
\hline Trauma & $2(14.3)$ & $0(0)$ & NA & NA & $0(0)$ & NA & NA & NA & NA \\
\hline Foreign body & $0(0)$ & $0(0)$ & NA & NA & $0(0)$ & NA & NA & NA & NA \\
\hline Dacryocystitis & $1(7.1)$ & $0(0)$ & NA & NA & $0(0)$ & NA & NA & NA & NA \\
\hline Dacryoadenitis & $2(14.3)$ & $0(0)$ & NA & NA & $0(0)$ & NA & NA & NA & NA \\
\hline Upper respiratory tract infection & $3(21.4)$ & $5(25.0)$ & NA & NA & $3(8.8)$ & NA & NA & NA & NA \\
\hline Uveitis & $0(0)$ & $0(0)$ & NA & NA & $0(0)$ & NA & NA & NA & NA \\
\hline Tumour & $0(0)$ & $0(0)$ & NA & NA & $0(0)$ & NA & NA & NA & NA \\
\hline \multicolumn{10}{|l|}{ Ophthalmologic signs } \\
\hline Lid swelling & $14(100.0)$ & $20(100.0)$ & NA & NA & NA & NA & NA & NA & NA \\
\hline Reduce visual acuity & $5(35.7)$ & NA & NA & NA & $11(32.4)$ & NA & NA & NA & NA \\
\hline Proptosis & $4(28.6)$ & $8(40.0)$ & NA & NA & $26(76.5)$ & NA & NA & $25(61.0)$ & NA \\
\hline Chemosis & $11(78.6)$ & $10(50.0)$ & NA & NA & $5(14.7)$ & NA & NA & NA & NA \\
\hline Ptosis & $7(50.0)$ & NA & NA & NA & NA & NA & NA & $30(73.2)$ & NA \\
\hline Ophthalmoplegia & $3(21.4)$ & $15(75.0)$ & NA & NA & $24(70.6)$ & NA & NA & $19(46.3)$ & NA \\
\hline None & $0(0)$ & NA & NA & NA & $0(0)$ & NA & NA & $11(26.8)$ & NA \\
\hline
\end{tabular}


Original Article

Table II. (Contd...)

\begin{tabular}{|c|c|c|c|c|c|c|c|c|c|}
\hline \multirow[t]{2}{*}{ Variable } & \multicolumn{9}{|c|}{ No. $(\%) /$ mean \pm standard deviation } \\
\hline & $\begin{array}{l}\text { Present } \\
\text { study }\end{array}$ & Yang et al ${ }^{(12)}$ & Liu et al $\mathbf{I}^{(15)}$ & Babu et al ${ }^{(13)}$ & $\begin{array}{l}\text { Ferguson } \\
\text { et } \mathrm{al}^{(4)}\end{array}$ & Atfeh \& Khali('(9) & Crosbie et al $\mathrm{I}^{(6)}$ & $\begin{array}{l}\text { Nageswaran } \\
\text { et al }{ }^{(5)}\end{array}$ & $\begin{array}{l}\text { Fanella } \\
\text { et al }\left.\right|^{(7)}\end{array}$ \\
\hline \multicolumn{10}{|l|}{ Visual acuity on presentation } \\
\hline $6 / 60$ & $1(7.1)$ & $0(0)$ & NA & NA & NA & NA & NA & NA & NA \\
\hline $6 / 21$ & $1(7.1)$ & $1(5.0)$ & NA & NA & NA & NA & NA & NA & NA \\
\hline $6 / 18$ & $0(0)$ & $0(0)$ & NA & NA & NA & NA & NA & NA & NA \\
\hline $6 / 12$ & $3(21.4)$ & $9(45.0)$ & NA & NA & NA & NA & NA & NA & NA \\
\hline $6 / 9$ & $1(7.1)$ & $0(0)$ & NA & NA & NA & NA & NA & NA & NA \\
\hline $6 / 6$ & $3(21.4)$ & $7(35.0)$ & NA & NA & NA & NA & NA & NA & NA \\
\hline Not cooperative & $5(35.7)$ & $2(10.0)$ & NA & NA & NA & NA & NA & NA & NA \\
\hline \multicolumn{10}{|l|}{ Final visual acuity } \\
\hline $6 / 21$ & $0(0)$ & $0(0)$ & NA & NA & NA & NA & NA & NA & NA \\
\hline $6 / 18$ & $0(0)$ & $0(0)$ & NA & NA & NA & NA & NA & NA & NA \\
\hline $6 / 12$ & $0(0)$ & $0(0)$ & NA & NA & NA & NA & NA & NA & NA \\
\hline $6 / 9$ & $1(7.1)$ & $0(0)$ & NA & NA & NA & NA & NA & NA & NA \\
\hline $6 / 6$ & $10(71.4)$ & $18(90.0)$ & NA & $3(60.0)$ & NA & NA & NA & NA & NA \\
\hline Not done & $3(21.4)$ & $2(10.0)$ & NA & NA & NA & NA & NA & NA & NA \\
\hline \multicolumn{10}{|l|}{ Length of hospitalisation (day) } \\
\hline All patients & $13.2 \pm 11.9$ & NA & NA & NA & 6.7 & $3.9 \pm 3.5$ & NA & $5.8 \pm 2.9$ & $7.0 \pm 2.7$ \\
\hline Medical only & $8.8 \pm 1.4^{*}$ & 6 & NA & NA & NA & NA & NA & $4.2 \pm 1.9$ & 3.9 \\
\hline Surgical intervention & $23.3 \pm 12.9^{+}$ & 11 & NA & NA & NA & NA & NA & $6.5 \pm 3.0$ & 6.1 \\
\hline \multicolumn{10}{|l|}{ Treatment } \\
\hline Medical only & $8(57.1)$ & $7(35.0)$ & $8(100.0)$ & $2(40.0)$ & $8(23.5)$ & NA & $5(16.7)$ & $12(29.3)$ & $30(78.9)$ \\
\hline Surgical intervention & $6(42.9)$ & $13(65.0)$ & NA & $3(60.0)$ & $26(76.5)$ & NA & $25(83.3)$ & $29(70.7)$ & $8(21.1)$ \\
\hline \multicolumn{10}{|l|}{ IV antibiotics } \\
\hline Augmentin & $4(28.6)$ & $8(40.0)$ & NA & $0(0)$ & NA & $36(90.0)$ & NA & $0(0)$ & $0(0)$ \\
\hline Cefuroxime & $0(0)$ & $0(0)$ & $0(0)$ & $0(0)$ & NA & $0(0)$ & $0(0)$ & $0(0)$ & $9(23.7)$ \\
\hline Cefotaxime & $0(0)$ & $0(0)$ & $0(0)$ & $0(0)$ & NA & $0(0)$ & $0(0)$ & $0(0)$ & $7(18.4)$ \\
\hline Amoxicillin & $0(0)$ & $0(0)$ & $1(12.5)$ & $0(0)$ & NA & NA & NA & $0(0)$ & $0(0)$ \\
\hline \multicolumn{10}{|l|}{3 antibiotics } \\
\hline Augmentin-metronidazole-ceftazidime & $2(14.3)$ & $0(0)$ & $0(0)$ & $0(0)$ & NA & NA & NA & $0(0)$ & $0(0)$ \\
\hline Augmentin-metronidazole-cefuroxime & $1(7.1)$ & $0(0)$ & $0(0)$ & $0(0)$ & NA & NA & NA & $0(0)$ & $0(0)$ \\
\hline Ceftazidime-metronidazole-vancomycin & $1(7.1)$ & $0(0)$ & $0(0)$ & $0(0)$ & NA & NA & NA & $0(0)$ & $0(0)$ \\
\hline \multicolumn{10}{|l|}{2 antibiotics } \\
\hline Ampicillin-cloxacillin & $0(0)$ & $3(15.0)$ & $0(0)$ & $0(0)$ & NA & NA & NA & $0(0)$ & $0(0)$ \\
\hline Augmentin-cloxacillin & $1(7.1)$ & $0(0)$ & $0(0)$ & $0(0)$ & NA & NA & NA & $0(0)$ & $0(0)$ \\
\hline Ceftriaxone-Augmentin & $1(7.1)$ & $0(0)$ & $0(0)$ & $0(0)$ & NA & NA & NA & $0(0)$ & $0(0)$ \\
\hline Ceftriaxone-cloxacillin & $1(7.1)$ & $8(40.0)$ & $0(0)$ & $0(0)$ & NA & NA & NA & $0(0)$ & $0(0)$ \\
\hline Ceftriaxone-metronidazole & $1(7.1)$ & $0(0)$ & $0(0)$ & $5(100.0)$ & NA & NA & NA & $0(0)$ & $0(0)$ \\
\hline
\end{tabular}


Table II. (Contd...)

\begin{tabular}{|c|c|c|c|c|c|c|c|c|c|}
\hline \multirow[t]{2}{*}{ Variable } & \multicolumn{9}{|c|}{ No. $(\%) / m e a n \pm$ standard deviation } \\
\hline & $\begin{array}{l}\text { Present } \\
\text { study }\end{array}$ & Yang et al ${ }^{(12)}$ & Liu et al ${ }^{(15)}$ & Babu et al ${ }^{(13)}$ & $\begin{array}{l}\text { Ferguson } \\
\text { et } \mathrm{al}^{\left({ }^{(4)}\right.}\end{array}$ & Atfeh \& Khali|(9) & Crosbie et al ${ }^{(6)}$ & $\begin{array}{l}\text { Nageswaran } \\
\text { et al }{ }^{(5)}\end{array}$ & $\begin{array}{l}\text { Fanella } \\
\text { et al }\left.\right|^{(7)}\end{array}$ \\
\hline Ceftriaxone-ceftazidime & $1(7.1)$ & $0(0)$ & $0(0)$ & $0(0)$ & NA & NA & NA & $0(0)$ & $0(0)$ \\
\hline Ceftazidime-metronidazole & $1(7.1)$ & $0(0)$ & $0(0)$ & $0(0)$ & NA & NA & NA & $0(0)$ & $0(0)$ \\
\hline Clindamycin-cephalosporin & $0(0)$ & $0(0)$ & $0(0)$ & $0(0)$ & NA & NA & NA & $0(0)$ & $8(21.1)$ \\
\hline Cloxacillin-cefotaxime & $0(0)$ & $0(0)$ & $0(0)$ & $0(0)$ & NA & NA & NA & $0(0)$ & $8(21.1)$ \\
\hline Ampicillin-sulbactam & $0(0)$ & $0(0)$ & $0(0)$ & $0(0)$ & NA & NA & NA & $19(46.3)$ & $0(0)$ \\
\hline Nafcillin-ceftrizoxime & $0(0)$ & $0(0)$ & $0(0)$ & $0(0)$ & NA & NA & NA & $11(26.8)$ & $0(0)$ \\
\hline \multicolumn{10}{|l|}{1 antibiotic } \\
\hline Vancomycin & $0(0)$ & $0(0)$ & $0(0)$ & $0(0)$ & NA & NA & NA & $0(0)$ & $0(0)$ \\
\hline Oxacillin & $0(0)$ & $0(0)$ & $4(50.0)$ & $0(0)$ & NA & NA & NA & $0(0)$ & $0(0)$ \\
\hline First-generation cephalosporin & $0(0)$ & $0(0)$ & $3(37.5)$ & $0(0)$ & NA & NA & NA & $0(0)$ & $0(0)$ \\
\hline Aminoglycoside & $0(0)$ & $0(0)$ & $3(37.5)$ & $0(0)$ & NA & NA & NA & $0(0)$ & $0(0)$ \\
\hline Antibiotic duration (day) & $10 \pm 12$ & NA & NA & NA & NA & NA & NA & $9.3 \pm 3.6$ & NA \\
\hline $1-7$ & $10(71.4)$ & NA & NA & NA & NA & NA & NA & NA & NA \\
\hline $8-14$ & $1(7.1)$ & NA & NA & NA & NA & NA & NA & NA & NA \\
\hline $15-30$ & $1(7.1)$ & NA & NA & NA & NA & NA & NA & NA & NA \\
\hline$>30$ & $2(14.3)$ & NA & NA & NA & NA & NA & NA & NA & NA \\
\hline \multicolumn{10}{|l|}{ Microorganism } \\
\hline \multicolumn{10}{|l|}{ Aerobes } \\
\hline a-/non-haemolytic streptococci & $0(0)$ & NA & $0(0)$ & NA & $0(0)$ & $8(30.8)^{*}$ & $0(0)$ & $7(17.1)$ & $1(2.6)$ \\
\hline Group A $\beta$-haemolytic streptococci & $2(14.3)$ & NA & $0(0)$ & NA & $3(8.8)$ & NA & $0(0)$ & $3(7.3)$ & $0(0)$ \\
\hline Streptococcus pyogenes & $0(0)$ & NA & $0(0)$ & NA & $0(0)$ & NA & $9(30.0)$ & $0(0)$ & $1(2.6)$ \\
\hline Streptococcus anginosus & $0(0)$ & NA & $0(0)$ & NA & $0(0)$ & NA & $3(10.0)$ & $0(0)$ & $0(0)$ \\
\hline Streptococcus pneumoniae & $0(0)$ & NA & $0(0)$ & NA & $1(2.9)$ & NA & $1(3.3)$ & $0(0)$ & $0(0)$ \\
\hline Staphylococcus aureus & $3(21.4)$ & $5(25.0)$ & $3(37.5)$ & NA & $5(14.7)$ & $8(30.8)^{*}$ & $0(0)$ & $3(7.3)$ & $1(2.6)$ \\
\hline Coagulase-negative Staphylococcus & $0(0)$ & $0(0)$ & $3(37.5)$ & NA & $0(0)$ & NA & $0(0)$ & $0(0)$ & $0(0)$ \\
\hline Methicillin-resistant Staphylococcus aureus & $1(7.1)$ & NA & $0(0)$ & NA & $0(0)$ & NA & $0(0)$ & $0(0)$ & $0(0)$ \\
\hline Burkholderia pseudomallei & $1(7.1)$ & $0(0)$ & $0(0)$ & NA & $0(0)$ & NA & $0(0)$ & $0(0)$ & $0(0)$ \\
\hline Haemophilus influenzae & $0(0)$ & $0(0)$ & $0(0)$ & NA & $0(0)$ & NA & $2(6.7)$ & $3(7.3)$ & $0(0)$ \\
\hline Group C $\beta$-haemolytic streptococci & $0(0)$ & $0(0)$ & $0(0)$ & NA & $0(0)$ & NA & $0(0)$ & $2(4.9)$ & $0(0)$ \\
\hline Eikenella corrodens & $0(0)$ & $0(0)$ & $0(0)$ & NA & $0(0)$ & NA & $0(0)$ & $2(4.9)$ & $0(0)$ \\
\hline Arcanobacterium haemolyticum & $0(0)$ & $0(0)$ & $0(0)$ & NA & $0(0)$ & NA & $0(0)$ & $1(2.4)$ & $0(0)$ \\
\hline Moraxella catarrhalis & $0(0)$ & $0(0)$ & $0(0)$ & NA & $0(0)$ & NA & $0(0)$ & $1(2.4)$ & $0(0)$ \\
\hline Pseudomonas aeruginosa & $0(0)$ & $1(5.0)$ & $0(0)$ & NA & $0(0)$ & NA & $0(0)$ & $0(0)$ & $0(0)$ \\
\hline Corynebacterium & $0(0)$ & $0(0)$ & $0(0)$ & NA & $0(0)$ & NA & $0(0)$ & $0(0)$ & $0(0)$ \\
\hline Anaerobes & $0(0)$ & $0(0)$ & $0(0)$ & NA & $4(11.8)$ & $2(7.7)^{*}$ & $0(0)$ & $8(19.5)$ & $0(0)$ \\
\hline No growth & $7(50.0)$ & $0(0)$ & NA & NA & NA & $14(53.8)^{*}$ & $8(26.7)$ & NA & $3(7.9)$ \\
\hline No specimen & $0(0)$ & NA & NA & NA & NA & NA & $3(10.0)$ & NA & NA \\
\hline
\end{tabular}

Values of $n$ have been omitted when they were not available. ${ }^{*} n=26$ (based on positive culture). $t n=8$. IV: intravenous; NA: not available; TWC: total white cell count 
Sinusitis and upper respiratory tract infection were the most common predisposing factors, occurring in $28.6 \%$ and $21.4 \%$ of our patients, respectively. These findings were consistent with Yang et al's report of a $30.0 \%$ incidence of sinusitis and $25.0 \%$ incidence of upper respiratory infection in their review involving 20 Singaporean children diagnosed with orbital cellulitis. ${ }^{(12)}$ In contrast, a very high incidence of sinusitis was reported among Australian and Canadian children (91.2\% and $94.7 \%$, respectively). ${ }^{(4,7)}$ Trauma was documented in $14.3 \%$ of the cases in this study. On the contrary, Ferguson et al and Yang et al reported no trauma in their studies. ${ }^{(4,12)}$

Final visual acuity of 6/9 (20/32) or better was documented in $78.6 \%$ of our patients. The remaining patients did not cooperate for the assessment of visual acuity during our assessment. A good final visual outcome of $90.0 \%$ was reported by Yang et al. ${ }^{(12)}$ Our patients stayed longer in the hospital (mean $13.2 \pm 11.9$ days) compared to other published data. (4,5-7,9) $^{-1}$ This was mainly due to difficult access to our hospital, poor clinical response to the initial antibiotic treatment and the necessity for a longer course of antibiotics. Duration of antibiotic course in our patients was fairly similar to that in the report by Nageswaran et al. ${ }^{(5)}$

Staphylococcus aureus (28.6\%) was the most common pathogen in our series. This observation was in keeping with studies conducted in Australia (14.7\%) and Singapore $(25.0 \%)^{(4,12)}$ Both Fanella et al and Atfeh and Khalil discovered that Staphylococcus aureus and non-haemolytic streptococci were the leading pathogen in their series. ${ }^{(7)}$ We also encountered cases infected with methicillin-resistant Staphylococcus aureus and Burkholderia pseudomallei. ${ }^{(16,17)}$ Both patients exhibited severe manifestations of infection, leading to a longer duration of antibiotics and length of hospitalisation. ${ }^{(16,17)}$ Infection due to Haemophilus influenzae was documented by Nageswaran et al $(7.3 \%)$ and Crosbie et al $(6.7 \%){ }^{(5,6)}$ Other infections were caused by Group C $\beta$-haemolytic streptococci, Eikenella corrodens, Arcanobacterium haemolyticum and Moraxella catarrhalis. ${ }^{(5)}$

Melioidosis is an uncommon cause for orbital cellulitis in children. Our patient in this study presented with bilateral orbital abscesses, subdural empyema and cavernous sinus thrombosis. ${ }^{(17)}$ A clinical assumption of melioidosis was made in this patient based on poor clinical response to conventional antibiotic therapy, borderline indirect haemagglutination assay titre and a favourable response after commencing ceftazidime treatment. It is pertinent to have a high index of suspicion for melioidosis when dealing with such patients who show poor response to conventional treatment in children, especially those living in endemic areas.

Broad choices for antibiotics were commenced based on clinical and laboratory results. Combinations of three antibiotics including Augmentin, metronidazole, ceftazidime, cefuroxime and vancomycin were prescribed for $28.6 \%$ of our patients. Dual combinations including Augmentin-cloxacillin, ceftriaxoneAugmentin, ceftriaxone-cloxacillin, ceftriaxone-metronidazole and ceftriaxone-ceftazidime were also started in our patients. An ampicillin-cloxacillin combination was used to treat $15.0 \%$ of patients in the study described by Yang et al. ${ }^{(12)}$ Patients treated with clindamycin-cephalosporin and cloxacillin-cefotaxime combinations represented $42.2 \%$ of the population in the study by Fanella et al. ${ }^{(7)}$ Nageswaran et al described that $46.3 \%$ of their patients were prescribed with an ampicillin-sulbactam combination, while another $26.8 \%$ was treated with nafcillinceftizoxime. ${ }^{(5)}$ The broad selection of antibiotics in the above studies was consistent with observations by Lee and Yen, who suggested that different communities have distinctive flora and varying resistance profiles. ${ }^{(18)}$

Surgical intervention was considered in $42.9 \%$ of our patients, while the others improved with medical treatment alone. Fanella et al reported that $21.1 \%$ of their patients underwent surgical interventions, ${ }^{(7)}$ which was inconsistent with the higher percentages of surgical interventions observed in Singapore (65\%), Australia (76.4\%), Scotland $(83.3 \%)$ and the United States $(70.7 \%)$. Indications for surgical intervention included larger abscesses with mass effect, poor clinical response to initial medical treatment and concurrent intracranial involvement. ${ }^{(12)}$ This phenomenon also suggests that ophthalmologists have different threshold levels for surgical interventions in managing paediatric orbital cellulitis. However, the information gathered from this study is limited to that from a single institution. Multicentre involvement would be beneficial to reflect a broader spectrum of the illness.

In conclusion, paediatric orbital cellulitis was more likely to affect young boys in our series. Methicillin-resistant Staphylococcus aureus and Burkholderia pseudomallei caused severe infection, while sinusitis and upper respiratory tract infection were the most common predisposing factors. A majority of the population improved with medical treatment alone. The findings from this study were slightly different from other published data on paediatric orbital cellulitis.

\section{REFERENCES}

1. Bedwell J, Bauman NM. Management of pediatric orbital cellulitis and abscess. Curr Opin Otolaryngol Head Neck Surg 2011; 19:467-73.

2. Rudloe TF, Harper MB, Prabhu SP, et al. Acute periorbital infections: who needs emergent imaging? Pediatrics 2010; 125:e719-26.

3. Baring DE, Hilmi OJ. An evidence based review of periorbital cellulitis. Clin Otolaryngol 2011; 36:57-64.

4. Ferguson MP, McNab AA. Current treatment and outcome in orbital cellulitis. Aust N Z J Ophthalmol 1999; 27:375-9.

5. Nageswaran S, Woods CR, Benjamin DK Jr, Givner LB, Shetty AK. Orbital cellulitis in children. Pediatr Infect Dis J 2006; 25:695-9.

6. Crosbie RA, Nairn J, Kubba H. Management of paediatric periorbital cellulitis: our experience of 243 children managed according to a standardised protocol 2012-2015. Int J Pediatric Otorhinolaryngol 2016; 87:134-8.

7. Fanella S, Singer A, Embree J. Presentation and management of pediatric orbital cellulitis. Can J Infect Dis Med Microbiol 2011; 22:97-100.

8. McKinley SH, Yen MT, Miller AM, Yen KG. Microbiology of pediatric orbital cellulitis. Am J Ophthalmol 2007; 144:497-501.

9. Atfeh MS, Khalil HS. Orbital infections: five-year case series, literature review and guideline development. J Laryngol Otol 2015; 129:670-6.

10. Elshafei AMK, Sayed MF, Abdallah RMA. Clinical profile and outcomes of management of orbital cellulitis in Upper Egypt. J Ophthalmic Inflamm Infect 2017; 7:8.

11. Seltz LB, Smith J, Durairaj VD, Enzenauer R, Todd J. Microbiology and antibiotic management of orbital cellulitis. Pediatrics 2011; 127:e566-72.

12. Yang $M$, Quah BL, Seah LL, Looi A. Orbital cellulitis in children-medical treatment versus surgical management. Orbit 2009; 28:124-36.

13. Babu PR, Prasad BD, Sowmya L, Sasanka KS. A review of preseptal and orbital cellulitis. J Evolution Med Dent Sci 2016; 5:1033-5. 
14. Pandian DG, Babu RK, Chaitra A, et al. Nine years' review on preseptal and orbital cellulitis and emergence of community-acquired methicillin-resistan Staphylococus aureus in a tertiary hospital in India. Indian J Ophthalmol 2011; 59:431-5.

15. Liu IT, Kao SC, Wang AG, et al. Preseptal and orbital cellulitis: a 10-year review of hospitalized patients. J Chin Med Assoc 2006; 69:415-22.

16. Chai-Lee T, Nadarajah S, Abdullah B, et al. Image-guided system endoscopic drainage of orbital abscess caused by methicillin-resistant Staphylococcus aureus in an infant. Int J Surg Case Rep 2017; 33:119-23.

17. Kogilavaani J, Shatriah I, Regunath K, Ahmad Helmy AK. Bilateral orbital abscesses with subdural empyema and cavernous sinus thrombosis due to meliodosis in a child. Asian Pac J Trop Dis 2014; 4 Suppl 2:S851-3.

18. Lee S, Yen MT. Management of preseptal and orbital cellulitis. Saudi J Ophthalmol 2011; 25:21-9. 